\title{
Neuropathy Severity at the Time of Oxaliplatin Treatment Alteration in Patients with Colon Cancer (Alliance A151912)
}

Daniel Hertz ( $\nabla$ dlhertz@med.umich.edu )

University of Michigan https://orcid.org/0000-0003-0501-1035

Travis J Dockter

Mayo Clinic College of Medicine and Science

Daniel V Satele

Mayo Clinic College of Medicine and Science

Charles L Loprinzi

Mayo Clinic College of Medicine and Science

Jennifer G Le-Rademacher

Mayo Clinic College of Medicine and Science

\section{Research Article}

Keywords: chemotherapy-induced peripheral neuropathy, colon cancer, treatment alteration, patient-reported outcomes, biomarkers, adverse event monitoring

Posted Date: April 5th, 2021

DOl: https://doi.org/10.21203/rs.3.rs-300187/v1

License: @ (1) This work is licensed under a Creative Commons Attribution 4.0 International License. Read Full License 


\section{Abstract}

Background: Clinical guidelines recommend altering chemotherapy treatment by decreasing, delaying, or discontinuing dosing in patients who are experiencing chemotherapy-induced peripheral neuropathy. There are few data available on the clinical use of treatment alteration including the severity of CIPN at the time of treatment alteration.

Methods: This was a retrospective analysis of patients receiving oxaliplatin on the NCCTG NO8CB trial. Neuropathy severity was assessed at each cycle by clinicians and patients. Patients were classified as 1) completed treatment without alteration, 2) dose reduction or delay due to neuropathy, 3) discontinuation due to neuropathy, or discontinuation for other toxicity (4), or another reason (5). Comparisons focused primarily on patients with alteration due to neuropathy (groups 2 and/or 3) compared with patients who completed treatment without alteration (group 1).

Results: In 350 participants, 135 (39\%) completed treatment without alteration, 70 (20\%) had a dose reduction or delay due to neuropathy, and $35(10 \%)$ discontinued early due to neuropathy. Clinician-assessed neuropathy severity was greater in patients at the time of dose reduction or delay compared with severity at the end of treatment in patients without alteration $(p<0.0001)$. Patient-reported neuropathy severity at cycle 4 was worse in patients who had an alteration as compared with patients who completed treatment without alteration $(p=0.017)$.

Conclusions: Treatment alterations due to neuropathy are common in patients receiving oxaliplatin for colon cancer and are associated with clinician-assessed neuropathy severity. Rapid increases in patient-reported neuropathy severity indicate a potential need for monitoring and intervention.

\section{Introduction}

One of the characteristic side effects of oxaliplatin, a standard component of adjuvant combination chemotherapy treatment of colorectal cancer ${ }^{1,2}$, is chemotherapy-induced peripheral neuropathy (CIPN). CIPN can present as sensory, motor, and/or painful symptoms in the hands and feet, which can lead to irreversible discomfort and dysfunction in some patients ${ }^{3}$. CIPN severity has historically been graded based on clinicianassessment using the National Cancer Institute Common Terminology Criteria for Adverse Events (NCl CTCAE) scale. ${ }^{4}$ CTCAE provides a uniform scale to grade toxicity severity from 0 (none) to 5 (causing death). There is an ongoing effort to supplement clinician-assessment in clinical research and practice with patient-reported toxicity collected via questionnaires, ${ }^{5,6}$ which can help detect toxicity earlier; such should, hopefully, improve patient quality of life. ${ }^{7}$ Patient-reported toxicities are particularly helpful when assessing subjective toxicities, such as CIPN. ${ }^{8}$ Multiple CIPN questionnaires have been developed, including the European Organisation for Research and Treatment of Cancer Quality of Life Questionnaire-Chemotherapy-Induced Peripheral Neuropathy 20 (EORTC QLQCIPN20) ${ }^{9}$.

Although CIPN questionnaires may improve monitoring and detection of neuropathy, there are limited effective interventions once CIPN is identified. Evidence-based practice guidelines from the American Society of Clinical Oncology (ASCO) do not recommend any pharmacological or behavioral intervention to prevent or treat CIPN, except for a recommendation to use duloxetine to treat painful CIPN. ${ }^{10}$ Instead, ASCO guidelines recommend 
considering altering neurotoxic chemotherapy treatment via dose reductions, delays, or early discontinuations in patients who are experiencing moderate or greater CIPN. Despite this recommendation in clinical practice guidelines, there is little known about the current practice of treatment alteration due to CIPN. Although studies have reported rates of oxaliplatin treatment alteration due to $\mathrm{CIPN},{ }^{11}, 12$ there is a lack of data on the severity of CIPN at the time of treatment alteration. This information is critical to develop evidence-based practice guidelines to inform when and how treatment should be altered, based on CIPN. The objective of this retrospective data analysis was to investigate, in patients receiving adjuvant treatment for colorectal cancer, the incidence of oxaliplatin treatment alterations, the CIPN severity at which treatment alterations were made, and whether there is any role for patient-reported CIPN in directing treatment alterations.

\section{Methods}

\section{Patients and Treatment}

Alliance for Clinical Trials in Oncology A151912 is a retrospective analysis conducted using data from North Central Cancer Treatment Group (NCCTG) study N08CB; NCCTG is now part of the Alliance. N08CB was a doubleblind randomized placebo-controlled phase III trial testing intravenous calcium and magnesium (CaMg) for prevention of oxaliplatin-induced neuropathy; that trial has been previously described in detail ${ }^{13}$. Briefly, N08CB enrolled patients with colon cancer, post-resection, who were scheduled to receive $85 \mathrm{mg} / \mathrm{m}^{2}$ oxaliplatin every 2 weeks for 12 cycles as part of fluorouracil, leucovorin, and oxaliplatin (FOLFOX) combination chemotherapy. Patients with prior neuropathy or who had previously received neurotoxic chemotherapy were ineligible to participate. All patients provided written IRB-approved, protocol-specific informed consent to participate, in accordance with federal and institutional guidelines. The study was performed in accordance with the ethical standards as laid down in the 1964 Declaration of Helsinki and its later amendments. Patients were randomized 1:1:1 to one of three arms receiving $\mathrm{CaMg}$ or placebo prior to and/or after treatment. Patient enrollment and matriculation through the study has been previously described and is illustrated in Figure 1. Of particular note for the current project, dose modifications (decreasing doses or discontinuing treatment) were not mandated by this protocol, although the protocol did suggest consideration of oxaliplatin dose reduction to $65 \mathrm{mg} / \mathrm{m}^{2}$ for patients who experienced persistent grade 2 sensory neuropathy that did not resolve within 2 weeks and oxaliplatin discontinuation for patients with persistent grade 3 sensory neuropathy.

\section{Neuropathy Data Collection}

CIPN data was collected prospectively within N08CB using both clinician-assessment and patient report. Clinician assessment was required at the start of each cycle using the National Cancer Institute Common Terminology Criteria for Adverse Events (NCl-CTCAE) version 4.0, employing the standard scale of 0-4 for both motor and sensory neuropathy. Clinicians were provided sample questions and answers with which to assign CTCAE grades (See Data Supplement ${ }^{13}$ ). CIPN was also collected before each cycle via patient-reported assessment using the European Organisation for Research and Treatment of Cancer Quality of Life Questionnaire-ChemotherapyInduced Peripheral Neuropathy 20 (EORTC QLQ-CIPN20) ${ }^{9}$. In the CIPN20, patients indicate the degree to which they experienced sensory ( 9 items), motor ( 8 items), and autonomic ( 3 items) symptoms during the past week using a scale of 1 ("not at all") to 4 ("very much"). The protocol analysis used the sensory subscale without the 
ototoxicity question (CIPN8), as the ototoxicity question has been found to not have adequate correlation with the

rest of the sensory subscale. ${ }^{14}$ Raw scores for the CIPN8 were scaled to 0-100, with higher scores indicating more CIPN.

\section{Oxaliplatin Treatment Alteration}

Oxaliplatin treatment data including the date and dose of treatment and reasons for treatment alteration was collected prospectively on N08CB. Treatment alteration was defined as any dose reduction ( $<81 \%$ of the planned dose), delay ( $>6$ days after planned dose), or discontinuation of oxaliplatin, irrespective of alterations to other chemotherapy and/or the study agent (CaMg or placebo). Information in the clinical research forms was then used to classify treatment alterations based on those attributed to CIPN, those attributed to another adverse event (i.e., non-CIPN adverse event or refused further treatment), and those attributed to any other reason (i.e., progression, death, or other/unknown). Based on the number and types of alterations, all patients included in this analysis were classified into five mutually exclusive categories: 1) completed treatment without oxaliplatin alteration, 2) oxaliplatin dose reduction or delay due to CIPN, 3) oxaliplatin discontinuation due to CIPN, 4) oxaliplatin discontinuation due to one or more other adverse events (AEs), or 5) oxaliplatin discontinuation for another reason.

\section{Statistical Analysis}

Since CaMg had no effect on CIPN in the primary analysis of N08CB ${ }^{13}$, this retrospective analysis (A151912) was conducted by pooling patients from all three study arms, similar to previously conducted secondary analyses of this cohort ${ }^{15}$. All five treatment alteration groups are reported throughout the manuscript, but comparisons focus primarily on patients with alteration due to CIPN (groups 2 and/or 3) compared with patients who completed treatment without an alteration (group 1). Analyses use the data (i.e., CIPN8, CTCAE, or oxaliplatin dose) at the time of first treatment alteration, or the data at the end of treatment in the group of patients who completed treatment without a treatment alteration (group 1). Demographic data were compared between patients with any alteration ( $n=105$, groups 2 and 3 ) and patients with no alteration ( $n=135$, group 1$)$. The CTCAE grade at the time of treatment alteration was compared among the five treatment alteration groups and between individual groups using chi-squared tests. The odds of any treatment alteration based on CTCAE grade was estimated using logistic regression (grade 2-3 vs 0-1). Median CIPN8 at the time of treatment alteration was compared across the five treatment alteration groups and between individual groups using Kruskal-Wallis tests. A post-hoc analysis of the change in CIPN8 from baseline to cycle 4, comparing patients who did not have any treatment alteration with patients who had alterations, was conducted using Kruskal-Wallis tests. All analyses were conducted by the Alliance Statistics and Data Center on the study database frozen on February 22, 2020 using SAS 9.4 with an uncorrected $a=0.05$. Therefore, all analyses should be considered exploratory.

\section{Results}

\section{Patients and Treatment Alterations}


This retrospective data analysis includes 350 N08CB participants who initiated oxaliplatin treatment and had any clinician-assessed or patient-reported CIPN data (Figure 1). Demographic data for this cohort has been previously reported $^{15}$, and is reported here stratified by the five treatment alteration groups (Table 1). There were 135 (39\%) patients who completed oxaliplatin without a treatment alteration, $70(20 \%)$ who had a dose reduction $(n=66)$ or delay $(n=4)$ due to CIPN, 35 (10\%) who had a dose discontinuation due to CIPN, and 110 who discontinued due to another $\operatorname{AE}(n=76,22 \%)$ or other reason $(n=34,10 \%)$. The group of patients who experienced alterations due to CIPN were younger (median 54 vs. $58, p=0.02)$ and were more likely to be female $(61 \%$ vs. $44 \%, 0.008)$ when compared to patients who completed treatment without alteration.

\section{Clinician-assessed CIPN at Time of Treatment Alteration}

Clinician-assessed CIPN severity by CTCAE grade at the time of treatment alteration, or end of treatment in the no alteration group, is reported in Table 2. The majority of patients who completed treatment without an alteration had grade 1 CIPN (68.1\%), whereas the majority of patients who had a treatment delay or reduction had grade 2 CIPN (60\%). Compared with CIPN severity in patients with no alteration, CIPN severity was greater in patients at the time of dose reduction or delay $(p<0.0001)$. In the logistic regression model, the risk of treatment alteration was significantly higher in patients with CTCAE grade 2-3 as compared to 0-1, when measured at the time of the dose change compared to other patients at a similar timeframe (OR: 4.40, 95\% Cl: 2.68,7.22, p<.0001).

\section{Patient-reported CIPN at Time of Treatment Alteration}

Patient-reported CIPN severity according to CIPN8 at the time of treatment alteration is reported in Table 2 and depicted in Figure 2. CIPN8 was not different when comparing patients who finished treatment without alteration to patients who had a dose reduction, delay or discontinuation, or to this group combined (all $p>0.05$, Table 2, Figure 2), understanding that differing amounts of oxaliplatin were received by these patient groups. Lower CIPN8 in patients with treatment alterations for other reasons should be interpreted with caution since these patients discontinued treatment early without experiencing CIPN. However, visual inspection of the change in CIPN8 from baseline stratified by treatment alteration group indicates greater early CIPN trajectory in patients who eventually had a treatment alteration (Figure 3). Based on this finding, a post hoc analysis was conducted of CIPN8 scores at cycle 4, which found greater increase in CIPN8 in patients who eventually had a reduction or delay as compared with patients who completed treatment without any alteration (median CIPN8 increase 11.5 vs. 7.7, $p=0.017)$.

\section{Discussion}

Alteration of neurotoxic chemotherapy treatment is the only intervention recommended for CIPN prevention or treatment in ASCO guidelines. ${ }^{10}$ However, there is a paucity of data on which to base treatment alteration decisions, including a lack of understanding of when alterations are used in practice. The current analysis of the real-world use of oxaliplatin for adjuvant treatment of colorectal cancer indicates that treatment alterations due to CIPN are relatively common. Rapid CIPN8 increases early in treatment are indicative of increased likelihood of a future need to alter treatment, indicating a potential use of early monitoring and intervention. 
In this cohort of patients receiving standard adjuvant oxaliplatin dosing for colorectal cancer, $>50 \%$ of patients had an oxaliplatin dose alteration and approximately 1 in 3 had an alteration due to CIPN. This incidence of CIPNrelated oxaliplatin treatment alteration is similar to the reported $13 \%-38 \%$ reported in other studies ${ }^{11,12}$. In the SCOT trial of patients $(n=3,044)$ with colorectal cancer receiving 3 months of adjuvant FOLFOX (oxaliplatin 85 $\mathrm{mg} / \mathrm{m}^{2}$ every 2 weeks) or CAPOX (oxaliplatin $130 \mathrm{mg} / \mathrm{m}^{2}$ every 3 weeks), 14\% of patients discontinued oxaliplatin and $30 \%$ had a dose reduction prior to completing treatment, though these were not all attributable to CIPN. ${ }^{16}$ Treatment alterations in that prospective clinical trial were mandated by the trial protocol, which may explain the much lower incidence of early discontinuation compared to the current cohort (42\% total, $10 \%$ due to CIPN). In the current study, patients with treatment alteration were more likely to be female and were younger. Increased treatment alterations in women is likely due to higher $\mathrm{CIPN}^{17}$, but could also be due to differences in treatment goals or communication with their oncologist. ${ }^{18}$ The association with age is somewhat surprising since older age is often reported to increase CIPN risk. ${ }^{19}$ It is possible that younger patients are altering treatment at lower CIPN severity to prevent irreversible toxicity, ${ }^{20}$ which would have more of an effect on a younger patient with longer life expectancy.

Investigating CIPN severity at the time of treatment alteration is ideally conducted in patient cohorts with prospective collection of toxicity data in which the protocol does not mandate treatment alteration, as is the case for N08CB. As expected, the incidence of treatment alteration increased more than 4-fold in patients with grade 2 or higher CTCAE CIPN. ${ }^{21}$ Surprisingly, this association was driven by dose decreases and delays, not early discontinuations, which is consistent with surveys of medical oncologists indicating that treatment discontinuation is the alteration of last resort for patients with the greatest CIPN severity. ${ }^{22}$ There was a patient in our cohort with treatment discontinuations despite grade 0 CIPN and a patient who completed treatment despite grade 3 CIPN, indicating there are substantial differences in acceptance of treatment discontinuation due to CIPN between oncologists and between patients. ${ }^{23}$ We previously reported a weak relationship between clinician- and patient-assessed CIPN in this cohort. ${ }^{24}$ These findings add further evidence that patient assessment is collecting data that are distinct from clinician assessment and decision making, which makes it challenging to define a clinically relevant change in patient-reported CIPN for use as a clinical trial endpoint. ${ }^{25,26}$

One potential use of patient reported outcomes supported by our data is to identify patients early in treatment who have a trajectory indicating future risk of treatment alteration. Another study previously reported that the severity of oxaliplatin-induced neuropathy midway through treatment is predictive of CIPN severity at the end of treatment, but did not include an assessment of treatment alteration. ${ }^{27}$ A modeling and simulation study using patient-reported CIPN during taxane treatment suggested that treatment alterations based on CIPN severity after 3 cycles could substantially reduce CIPN severity at the end of 6 cycles of treatment. ${ }^{28}$ However, prospective clinical trials are needed to demonstrate the benefit and understand the risks of decreasing oxaliplatin dosing based on early indicators of CIPN. Prospective testing of a similar strategy of oxaliplatin treatment alteration informed by early changes in quantitative sensory testing did not reduce CIPN. ${ }^{29}$ Perhaps, this different outcome between patients receiving paclitaxel and oxaliplatin might be related to the coasting phenomenon seen with oxaliplatin whereby, on average, CIPN worsens for 3 months after oxaliplatin is stopped.

One of the major strengths of this study was the use of prospectively collected data of oxaliplatin dosing, CIPN assessment by patients and clinicians, and reasons for oxaliplatin alteration, including CIPN. Importantly, this trial did not mandate treatment alteration, providing a unique opportunity to characterize real-world practice of 
treatment alteration and the corresponding CIPN severity, as defined by clinician and patient assessment. There are also limitations of this study that should be considered, including the retrospective data analysis, which can allow for unforeseen sources of bias. There is always the possibility that some data were missing or incorrect, including in our retrospective assignment of treatment alteration categories. Additionally, these results may only be representative of adjuvant oxaliplatin treatment for colorectal cancer, as treatment alteration decisions are likely to be dependent on the treatment setting. Oxaliplatin has a well-known coasting effect ${ }^{30}$, in which CIPN severity continues to increase after treatment discontinuation, meaning that clinicians may be making treatment alterations based on their expectation of greater CIPN severity in the future and not the present severity.

In conclusion, this analysis provides further evidence that oxaliplatin treatment alteration due to CIPN is common and is most strongly associated to the clinician's assessment of severity, and that rapid early increases in patientreported severity may be indicative of high risk of future treatment alteration. Additional research, including prospective clinical trials, is needed to understand the benefits and risks of altering treatment in order to develop evidence-based guidelines for when and how treatment alteration should be used to prevent long-term CIPN and maximize treatment outcomes in patients with cancer.

\section{Disclosures And Declarations}

Funding: Research reported in this publication was supported by the National Cancer Institute of the National Institutes of Health under the Award Number UG1CA189823 (Alliance for Clinical Trials in Oncology NCORP Grant) and UG1CA232760. https://acknowledgments.alliancefound.org. The content is solely the responsibility of the authors and does not necessarily represent the official views of the National Institutes of Health.

Conflicts of Interest/Competing interests: The authors have no relevant employment to disclose. The authors have declared all relevant financial interests including consulting fees from PledPharma, Disarm Therapeutics, Asahi Kasei, Metys Pharmaceuticals, NKMax, Novartis, HengRui, Nruo Bio, Osmol Therapeutics Inc, and Grunenthal to Dr. Loprinzi for research related to chemotherapy-induced peripheral neuropathy. The authors have also received funding from the Alliance for Clinical Trials in Oncology (ACTION) for travel related to attending meetings.

Availability of Data and Material: The data used in this analysis is maintained by the Alliance for Clinical Trials in Oncology. Data will be made available upon reasonable request to the corresponding author.

Code Availability: The code used in this analysis will be made available upon reasonable request to the corresponding author.

Authors' contributions: DLH, CLL, JGL contributed to conception and design. DVS, TJD, and JGL contributed to data analysis. All authors contributed to interpretation of data. DLH drafted the manuscript. All co-authors revised the manuscript and approved of the version to be published.

Ethics approval: The study was performed in accordance with the ethical standards as laid down in the 1964 Declaration of Helsinki and its later amendments.

Clinical Trial Registration: The parent clinical trial within which this retrospective analysis was conducted was registered within clinicaltrials.gov Identifier: NCT01099449 (NCCTG N08CB). 
Consent to Participate: All patients provided written IRB-approved, protocol-specific informed consent to participate, in accordance with federal and institutional guidelines.

Consent for Publication: All patients provided written IRB-approved, protocol-specific informed consent to participate, in accordance with federal and institutional guidelines.

\section{Tables}

Table 1. Demographic and Treatment Information for Patients Included in the Analysis

\begin{tabular}{|c|c|c|c|c|c|c|}
\hline & $\begin{array}{l}\text { No } \\
\text { Alterations } \\
(\mathrm{N}=135)\end{array}$ & $\begin{array}{l}\text { Reductions } \\
\text { or delays } \\
(\mathrm{N}=70)\end{array}$ & $\begin{array}{l}\text { Discontinuation } \\
\text { for Neuropathy } \\
(\mathrm{N}=35)\end{array}$ & $\begin{array}{l}\text { Discontinuation } \\
\text { for Other } A E \\
(\mathrm{~N}=76)\end{array}$ & $\begin{array}{l}\text { Discontinuation } \\
\text { for Other } \\
(\mathrm{N}=34)\end{array}$ & $\begin{array}{l}\mathrm{p}- \\
\text { value }\end{array}$ \\
\hline $\begin{array}{l}\text { Age } \\
\text { (years) }\end{array}$ & & & & & & 0.025 \\
\hline $\begin{array}{l}\text { Median } \\
\text { (range) }\end{array}$ & $58(24-83)$ & $51(27-77)$ & $56(39-79)$ & $58(29-83)$ & $63(28-83)$ & \\
\hline $\begin{array}{l}\text { Gender, } \mathrm{n} \\
(\%)\end{array}$ & & & & & & 0.008 \\
\hline Female & $59(43.7 \%)$ & 41 (58.6\%) & $23(65.7 \%)$ & $44(57.9 \%)$ & $16(47.1 \%)$ & \\
\hline Male & 76 (56.3\%) & 29 (41.4\%) & $12(34.2 \%)$ & $32(42.1 \%)$ & $18(52.9 \%)$ & \\
\hline $\begin{array}{l}\text { Race, } n \\
(\%)\end{array}$ & & & & & & 0.22 \\
\hline White & $\begin{array}{l}106 \\
(78.5 \%)\end{array}$ & 64 (91.4\%) & $30(85.7 \%)$ & 69 (90.8\%) & $28(82.4 \%)$ & \\
\hline Black & $22(16.3 \%)$ & $6(8.6 \%)$ & $4(11.4 \%)$ & $6(7.9 \%)$ & $3(8.8 \%)$ & \\
\hline $\begin{array}{l}\text { Other or } \\
\text { Unknown }\end{array}$ & $7(5.2 \%)$ & $0(0.0 \%)$ & $1(2.9 \%)$ & $1(1.3 \%)$ & $3(8.8 \%)$ & \\
\hline $\begin{array}{l}\text { Stage, } n \\
(\%)\end{array}$ & & & & & & 0.11 \\
\hline II & 27 (20.0\%) & 7 (10.0\%) & $6(17.1 \%)$ & $19(25.0 \%)$ & 7 (20.6\%) & \\
\hline III & 96 (71.1\%) & 61 (87.1\%) & $26(74.3 \%)$ & 55 (72.4\%) & $24(70.6 \%)$ & \\
\hline IV & 12 (8.9\%) & $2(2.9 \%)$ & $3(8.6 \%)$ & $2(2.6 \%)$ & $3(8.8 \%)$ & \\
\hline
\end{tabular}

Data reported as $\mathrm{n}(\%)$ or median (range).

Table 2: Cumulative Oxaliplatin Dosing, CTCAE, and CIPN8 at Time of Alteration 


\begin{tabular}{|c|c|c|c|c|c|c|}
\hline & $\begin{array}{l}\text { No } \\
\text { Alterations } \\
(\mathrm{N}=135)\end{array}$ & $\begin{array}{l}\text { Reductions } \\
\text { or delays } \\
(\mathrm{N}=70)\end{array}$ & $\begin{array}{l}\text { Discontinuation } \\
\text { for Neuropathy } \\
(\mathrm{N}=35)\end{array}$ & $\begin{array}{l}\text { Discontinuation } \\
\text { for Other } A E \\
(\mathrm{~N}=76)\end{array}$ & $\begin{array}{l}\text { Discontinuation } \\
\text { for Other } \\
(\mathrm{N}=34)\end{array}$ & $\begin{array}{l}\text { p- } \\
\text { value* }\end{array}$ \\
\hline \multicolumn{7}{|l|}{$\begin{array}{l}\text { Cumulative } \\
\text { Oxaliplatin }\end{array}$} \\
\hline Cycles & $12(12-12)$ & $7(3-12)$ & $10(4-11)$ & $6(1-11)$ & $6.5(1-11)$ & $<0.0001$ \\
\hline Dose (mg) & $\begin{array}{l}1860 \\
(1230- \\
2760)\end{array}$ & $\begin{array}{l}980(260- \\
1980)\end{array}$ & $\begin{array}{l}1570(426- \\
2108)\end{array}$ & $885(150-2360)$ & 918 (139-1969) & $<0.0001$ \\
\hline $\begin{array}{l}\text { CIPN } \\
\text { CTCAE }\end{array}$ & & & & & & $<0.0001$ \\
\hline Grade 0 & 12 (8.9\%) & 2 (2.9\%) & 1 (2.9\%) & 23 (30.3\%) & $6(17.6 \%)$ & \\
\hline Grade 1 & $92(68.1 \%)$ & 24 (34.3\%) & $23(65.7 \%)$ & $42(55.3 \%)$ & $21(61.8 \%)$ & \\
\hline Grade 2 & $30(22.2 \%)$ & $42(60.0 \%)$ & $10(28.6 \%)$ & 8 (10.5\%) & 7 (20.6\%) & \\
\hline Grade 3 & $1(0.7 \%)$ & 2 (2.9\%) & $1(2.9 \%)$ & $3(3.9 \%)$ & $0(0.0 \%)$ & \\
\hline \multicolumn{7}{|l|}{ CIPN8 } \\
\hline Mean (SD) & $\begin{array}{l}32.6 \\
(17.91)\end{array}$ & $\begin{array}{l}33.4 \\
(16.35)\end{array}$ & $28.9(16.26)$ & $22.5(14.29)$ & $17.4(9.62)$ & NR \\
\hline $\begin{array}{l}\text { Median } \\
\text { (Range) }\end{array}$ & $\begin{array}{l}26.9(7.7- \\
92.3)\end{array}$ & $\begin{array}{l}30.8(7.7- \\
88.5)\end{array}$ & $23.1(7.7-65.4)$ & $19.2(7.7-73.1)$ & $15.4(7.7-46.2)$ & 0.91 \\
\hline
\end{tabular}

Dose reported in mean (standard deviation) and cycles reported in median (range)

*P-value comparing patients that completed treatment without alteration (column $1, n=135$ ) with a combination of patients that had any alteration (i.e., reduction, delay, or discontinuation) due to neuropathy (columns 2 and 3 , $n=105)$

CTCAE: National Cancer Institute Common Terminology Criteria for Adverse Events

NR: not reported due to skewness in the CIPN8 data. See comparison of CIPN8 medians.

\section{References}

1. Referenced from the NCCN Clinical Practice Guidelines in Oncology (NCCN Guidelines ${ }^{\circledR}$ ) for Colon Cancer V.2.2019. @ National Comprehensive Cancer Network, Inc 2019. All rights reserved. Accessed [June 27, 2019]. To view the most recent and complete version of the guideline, go online to www.nccn.org. NATIONAL COMPREHENSIVE CANCER NETWORK $®$, NCCN ${ }^{\circ}$, NCCN GUIDELINES ${ }^{\circledR}$, and all other NCCN Content are trademarks owned by the National Comprehensive Cancer Network, Inc.

2. Referenced from the NCCN Clinical Practice Guidelines in Oncology (NCCN Guidelines ${ }^{\circledR}$ ) for Rectal Cancer V.2.2019. () National Comprehensive Cancer Network, Inc 2019. All rights reserved. Accessed [July 16, 2019]. To view the most recent and complete version of the guideline, go online to www.nccn.org. 
NATIONAL COMPREHENSIVE CANCER NETWORK®, NCCN®, NCCN GUIDELINES ${ }^{\circledR}$, and all other NCCN Content are trademarks owned by the National Comprehensive Cancer Network, Inc.

3. Yoshino T, Kotaka M, Shinozaki K, et al. JOIN trial: treatment outcome and recovery status of peripheral sensory neuropathy during a 3-year follow-up in patients receiving modified FOLFOX6 as adjuvant treatment for stage II/III colon cancer. Cancer Chemother Pharmacol. 2019;84:1269-1277. doi: 1210.1007/s0028000019-03957-00285. Epub 02019 Sep 00223.

4. Common Terminology Criteria for Adverse Events (CTCAE) Version 4.0: U.S. Department of Health and Human Services, National Institutes of Health, National Cancer Insitute; 2010.

5. Basch E, Dueck AC, Rogak LJ, et al. Feasibility assessment of patient reporting of symptomatic adverse events in multicenter cancer clinical trials. JAMA Oncology. 2017.

6. Basch E, Jia X, Heller G, et al. Adverse symptom event reporting by patients vs clinicians: relationships with clinical outcomes. J Natl Cancer Inst. 2009;101:1624-1632.

7. Basch E, Deal AM, Dueck AC, et al. Overall Survival Results of a Trial Assessing Patient-Reported Outcomes for Symptom Monitoring During Routine Cancer Treatment. JAMA. 2017;318:197-198.

8. Di Maio M, Basch E, Bryce J, Perrone F. Patient-reported outcomes in the evaluation of toxicity of anticancer treatments. Nat Rev Clin Oncol. 2016;13:319-325.

9. Postma TJ, Aaronson NK, Heimans JJ, et al. The development of an EORTC quality of life questionnaire to assess chemotherapy-induced peripheral neuropathy: the QLQ-CIPN20. Eur J Cancer. 2005;41:1135-1139.

10. Loprinzi CL, Lacchetti C, Bleeker J, et al. Prevention and Management of Chemotherapy-Induced Peripheral Neuropathy in Survivors of Adult Cancers: ASCO Guideline Update. J Clin Oncol. 2020;14:01399.

11. Beijers AJ, Mols F, Tjan-Heijnen VC, Faber CG, van de Poll-Franse LV, Vreugdenhil G. Peripheral neuropathy in colorectal cancer survivors: the influence of oxaliplatin administration. Results from the population-based PROFILES registry. Acta Oncol. 2015;54:463-469. doi: 410.3109/0284186X.0282014.0980912. Epub 0282014 Nov 0284124.

12. Ali R, Baracos VE, Sawyer MB, et al. Lean body mass as an independent determinant of dose-limiting toxicity and neuropathy in patients with colon cancer treated with FOLFOX regimens. Cancer Med. 2016;5:607-616. doi: 610.1002/cam1004.1621. Epub 2016 Jan 1027.

13. Loprinzi CL, Qin R, Dakhil SR, et al. Phase III randomized, placebo-controlled, double-blind study of intravenous calcium and magnesium to prevent oxaliplatin-induced sensory neurotoxicity (N08CB/Alliance). J Clin Oncol. 2014;32:997-1005.

14. Lavoie Smith EM, Barton DL, Qin R, Steen PD, Aaronson NK, Loprinzi CL. Assessing patient-reported peripheral neuropathy: the reliability and validity of the European Organization for Research and Treatment of Cancer QLQ-CIPN20 Questionnaire. Quality of life research : an international journal of quality of life aspects of treatment, care and rehabilitation. 2013;22:2787-2799.

15. Pachman DR, Qin R, Seisler DK, et al. Clinical Course of Oxaliplatin-Induced Neuropathy: Results From the Randomized Phase III Trial N08CB (Alliance). J Clin Oncol. 2015;33:3416-3422.

16. Iveson TJ, Kerr RS, Saunders MP, et al. 3 versus 6 months of adjuvant oxaliplatin-fluoropyrimidine combination therapy for colorectal cancer (SCOT): an international, randomised, phase 3, non-inferiority trial. Lancet Oncol. 2018;19:562-578. doi: 510.1016/S1470-2045(1018)30093-30097. 
17. Wagner AD, Grothey A, Andre T, et al. Sex and adverse events of adjuvant chemotherapy in colon cancer: an analysis of 34,640 patients in the ACCENT database. J Natl Cancer Inst. 2020;24.

18. Knoerl R, Smith EML, Han A, Doe A, Scott K, Berry DL. Characterizing patient-clinician chemotherapy-induced peripheral neuropathy assessment and management communication approaches. Patient Educ Couns. 2019;102:1636-1643. doi: 1610.1016/j.pec.2019.1604.1012. Epub 2019 Apr 1639.

19. Chan A, Hertz DL, Morales M, et al. Biological predictors of chemotherapy-induced peripheral neuropathy (CIPN): MASCC neurological complications working group overview. Support Care Cancer. 2019;27:37293737.

20. Bandos H, Melnikow J, Rivera DR, et al. Long-term Peripheral Neuropathy in Breast Cancer Patients Treated With Adjuvant Chemotherapy: NRG Oncology/NSABP B-30. J Nat/ Cancer Inst. 2018;110.

21. Beutler AS, Majithia N, Loprinzi CL. The past and future of 'reported outcomes' in studies on chemotherapy neuropathy. Ann Oncol. 2017;28:2631-2632.

22. Kuroi K, Shimozuma K, Ohashi Y, et al. A questionnaire survey of physicians' perspectives regarding the assessment of chemotherapy-induced peripheral neuropathy in patients with breast cancer. Jpn J Clin Oncol. 2008;38:748-754.

23. Salgado TM, Quinn CS, Krumbach EK, et al. Reporting of paclitaxel-induced peripheral neuropathy symptoms to clinicians among women with breast cancer: a qualitative study. Support Care Cancer. 2020;28:4163-4172. doi: 4110.1007/s00520-00019-05254-00526. Epub 02020 Jan 00522.

24. Le-Rademacher J, Kanwar R, Seisler D, et al. Patient-reported (EORTC QLQ-CIPN20) versus physician-reported (CTCAE) quantification of oxaliplatin- and paclitaxel/carboplatin-induced peripheral neuropathy in NCCTG/Alliance clinical trials. Support Care Cancer. 2017;25:3537-3544.

25. Draak THP, de Greef BTA, Faber CG, Merkies ISJ. The minimum clinically important difference: which direction to take. Eur J Neurol. 2019;26:850-855. doi: 810.1111/ene.13941. Epub 12019 Mar 13925.

26. Hertz DL. Concerns regarding use of patient-reported outcomes in biomarker studies of chemotherapyinduced peripheral neuropathy. Pharmacogenomics J. 2019;19:411-416.

27. Velasco R, Bruna J, Briani C, et al. Early predictors of oxaliplatin-induced cumulative neuropathy in colorectal cancer patients. J Neurol Neurosurg Psychiatry. 2014;85:392-398. doi: 310.1136/jnnp-2013-305334. Epub 302013 Jun 305329.

28. Sharma MR, Mehrotra S, Gray E, et al. Personalized Management of Chemotherapy-Induced Peripheral Neuropathy Based on a Patient Reported Outcome: CALGB 40502 (Alliance). J Clin Pharmacol. 2020;60:444452. doi: 410.1002/jcph.1559. Epub 2019 Dec 1004.

29. Delmotte JB, Beaussier $\mathrm{H}$, Auzeil $\mathrm{N}$, et al. Is quantitative sensory testing helpful in the management of oxaliplatin neuropathy? a two-year clinical study. Cancer Treat Res Commun. 2018;17:3136.:10.1016/j.ctarc.2018.1010.1002. Epub 2018 Oct 1010.

30. Albany C, Dockter T, Wolfe E, et al. Cisplatin-associated neuropathy characteristics compared with those associated with other neurotoxic chemotherapy agents (Alliance A151724). Support Care Cancer. 2020;4:020-05543.

\section{Figures}




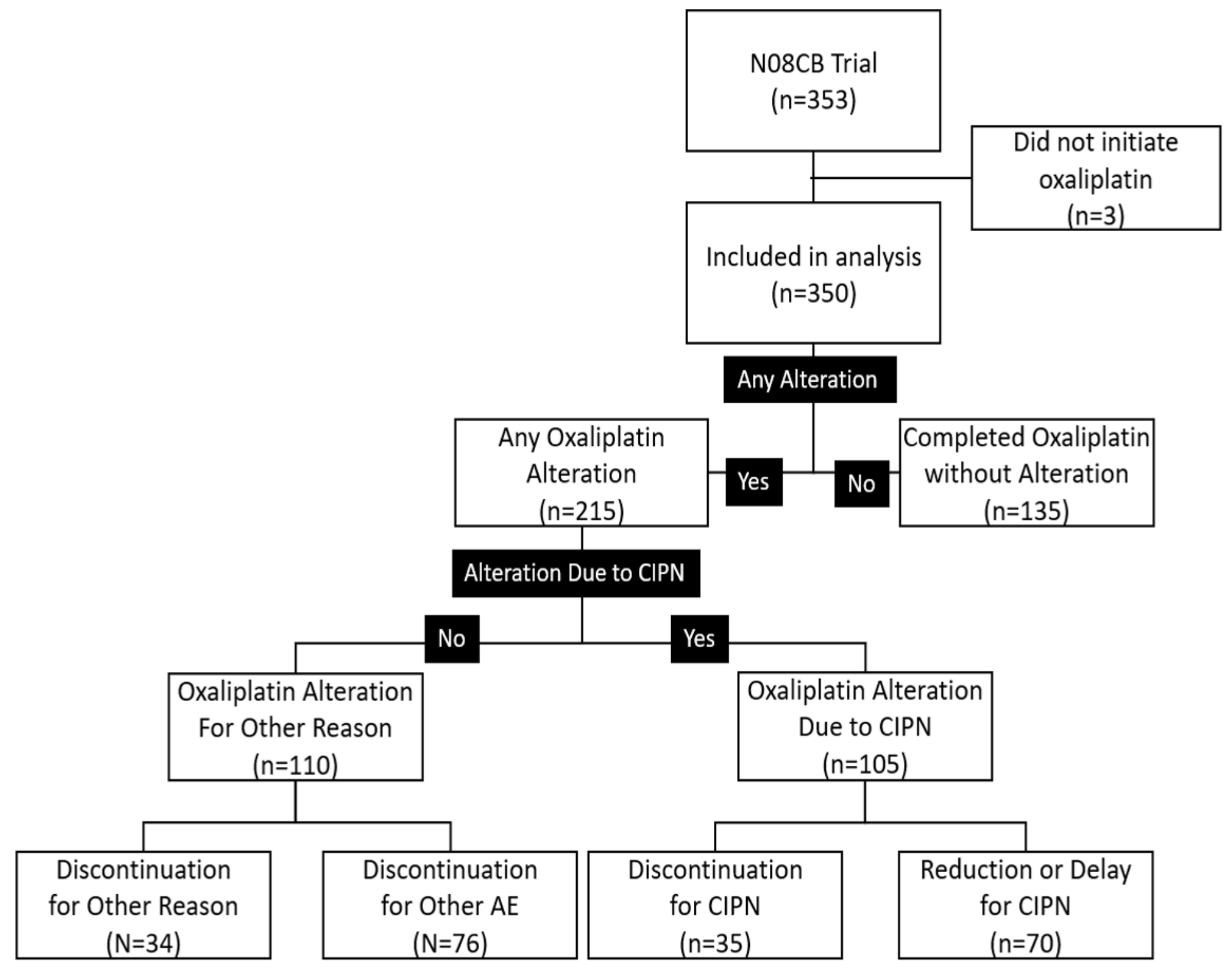

\section{Figure 1}

CONSORT Diagram: Patients from N08CB were categorized into five treatment alteration groups. The main comparisons of interest were those patients who completed oxaliplatin treatment with no alteration $(n=135)$ compared to patients who had a reduction or delay for CIPN $(n=70)$ and/or patients who had a discontinuation for CIPN (n=35). Patients who discontinued for other reasons were reported but were not included in most analyses because they are less informative. Discontinuation for Other AE included: Refused Further Treatment $(n=28)$ or non-CIPN Adverse Event $(n=48)$. Discontinuation for Other Reason included: Disease Progression $(n=3)$, Switch to Alternate Treatment $(n=5)$, Other Medical Problems $(n=6)$, Died on Study $(n=3)$, or Other $(n=9)$ 


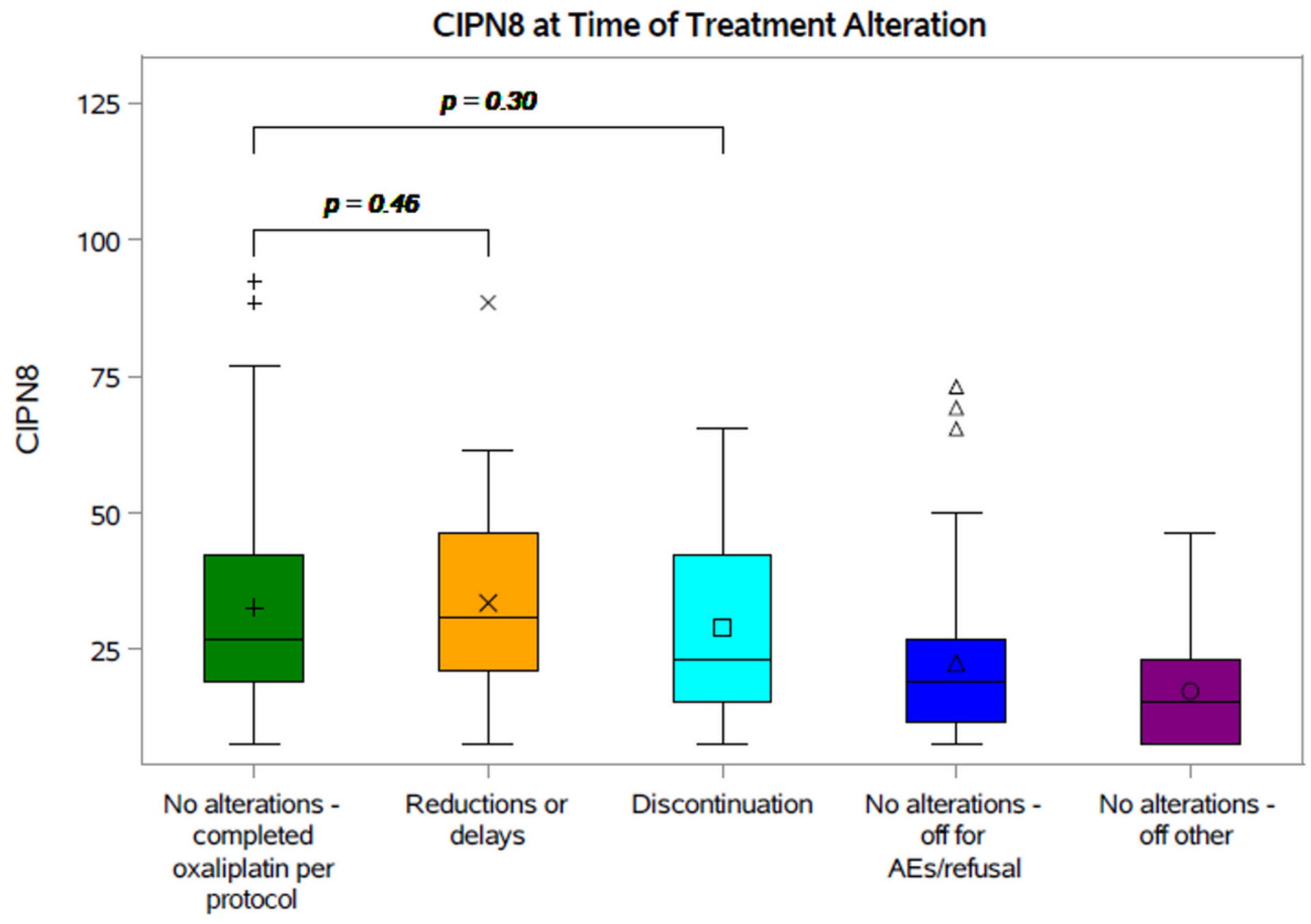

Neurotoxicity and Study Completion status

Figure 2

CIPN8 at Time of Treatment Alteration. Patient-reported CIPN, defined by the sensory CIPN8 (range 0-100, higher scores indicating greater CIPN), at the time of qualifying event stratified by the five treatment alteration groups. The three groups of interest (groups 1-3 from left) were not statistically different. 


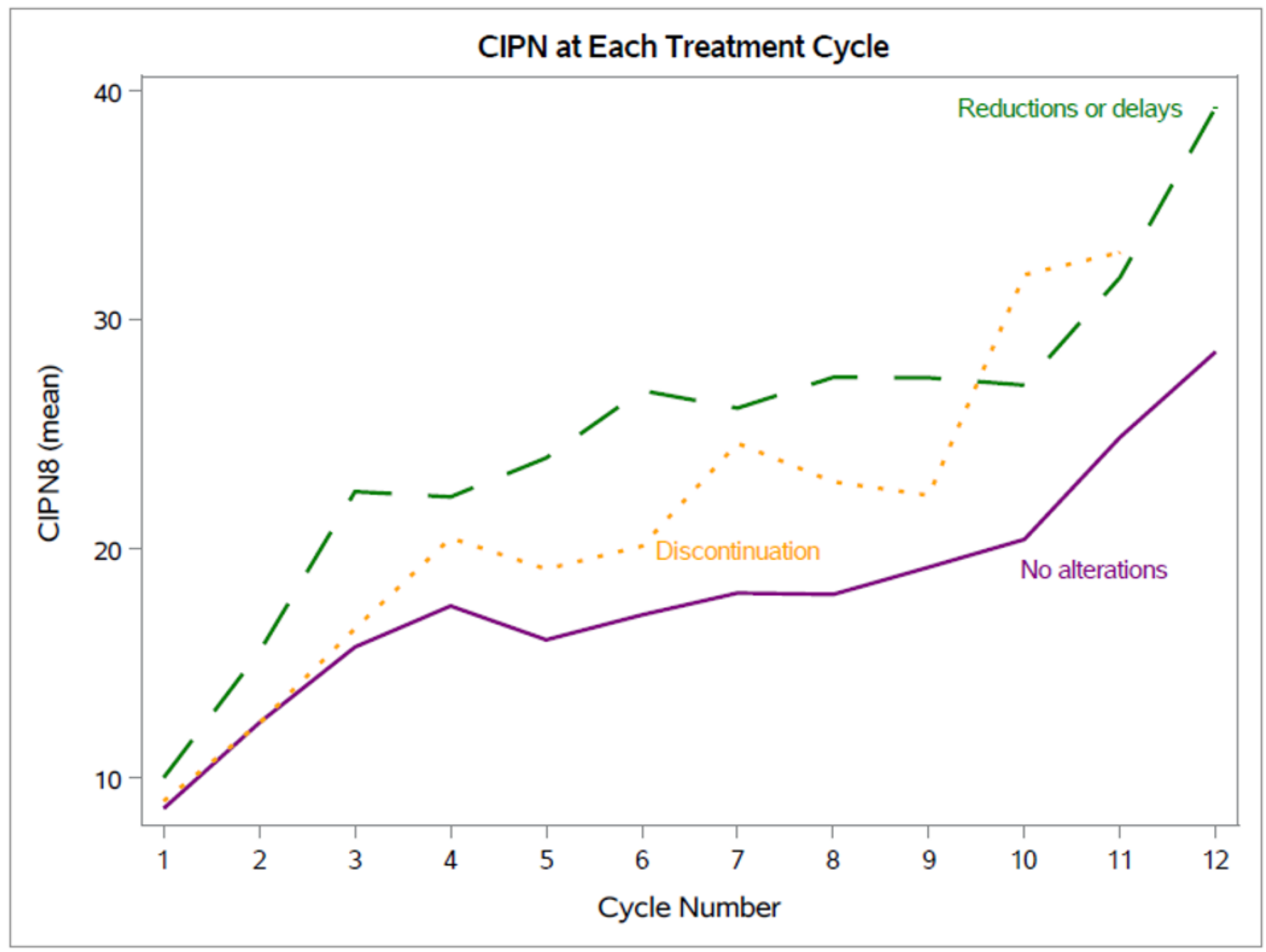

\begin{tabular}{|l|r|r|r|r|r|r|r|r|r|r|r|r|}
\hline & C1 & C2 & C3 & C4 & C5 & C6 & C7 & C8 & C9 & C10 & C11 & C12 \\
\hline No Alterations ( $\mathrm{N}=135)$ & 132 & 125 & 130 & 127 & 126 & 129 & 126 & 129 & 126 & 130 & 128 & 118 \\
\hline Reductions or delays (N=70) & 70 & 68 & 67 & 67 & 64 & 66 & 65 & 60 & 55 & 49 & 42 & 41 \\
\hline Discontinuation (N=35) & 33 & 32 & 34 & 34 & 34 & 26 & 30 & 27 & 21 & 19 & 7 & 0 \\
\hline
\end{tabular}

\section{Figure 3}

Trajectory of CIPN8 During Treatment. Mean patient-reported CIPN, defined by the sensory CIPN8 (range 0-100 higher scores indicating greater (IPN) at each treatment cycle, is plotted for each of the treatment alteration groups. Patients who eventually have oxaliplatin reductions or delays (green) or early discontinuation (yellow) due to CIPN have faster increases in CIPN8 than patients who complete treatment without any alteration (purple). 